\title{
Detection of Streptococcus mutans in Plaque Samples by the Direct Fluorescent Antibody Test
}

WALTER J. LOESCHE and ELLA GRENIER

Dental Research Institute and Department of Oral Biology, University of

Michigan, School of Dentistry, Ann Arbor, Michigan 48104, USA

Culture methods traditionally have been used to isolate and identify bacterial species in clinical specimens. Culture methods are difficult when the specimen is as bacteriologically complex as dental plaque ${ }^{1}$ and when the organism in question, such as Streptococcus mutans, is present in modest numbers. The fluorescent antibody test (FAT) has been suggested as a potentially simpler method of detecting $S$ mutans in plaque samples. ${ }^{2,3}$ In the most commonly described procedure, plaque is cultured in broth media and after growth occurs, smears are prepared and stained with fluorescein-conjugated antiserums against the various $S$ mutans serotypes. 4,5 This procedure detects whether $S$ mutans is present, but provides no information concerning the proportional levels of $S$ mutans in the plaque samples. Culture studies indicate that the percentages of $S$ mutans in plaque samples show a greater association with dental decay than does the presence of $S$ mutans,6,7 FATs performed on broth cultures of plaque 5,8 or upon isolates of $S m u$ $\operatorname{tans}^{9,10}$ show that serotype $c$ is the most prevalent serotype in human plaque. However, direct FAT performed on smears of plaque collected from an adolescent male population exhibiting active caries (that is, an average of nine teeth with open lesions) showed serotype $d$ to be the most common serotype, and that serotype $c$ was rarely present. ${ }^{11}$ This discrepancy indicates that the human populations sampled had different $S$ mutans serotypes, that the fluorescein-conjugated antiserums used were of different sensitivities, that the anti- $d$ serum reacts with organisms present in the smear that do not grow up in the broth, that the broth or agar cultures

\footnotetext{
This work was supported by US Public Health Grants Nos. DE-02731, DE-03011, and DE-03423.
}

select for serotype $c$ strains, and that serotype $c$ strains are masked in the plaque in such a way that they did not stain with the FAT reagents. In the present investigation, the same plaque samples were cultured and also examined by the FAT before and after growth in broth. In addition, the FAT and culture studies were used to monitor the efficacy of topical treatment with fluoride or kanamycin as a means of reducing the plaque levels of $S$ mutans.

\section{Materials and Methods}

Preparation of Fluorescent antibody REAGENTS.-Representative strains of $S$ mutans serotypes $b, c, d$, and $e^{3}$ were grown in a medium containing equal parts of Spirolate and brain-heart infusion broths (Bioquest) to which $0.5 \%$ yeast extract was added. The medium will be designated hereafter as SBY broth. A conjugate against AHT also was used so as to give a serum against serotype $a$. However, subsequent biochemical tests ${ }^{12}$ showed that this strain of AHT belonged to serotype $d$, so that no data were obtained for serotype $a$ cells in the present investigation. After 48 hours of growth, the cells were harvested by centrifugation and incubated with $0.4 \%$ Formalin for two days at $37 \mathrm{C}$. The cells with Formalin then were washed, diluted to a no. 5 MacFarland standard, and used as antigens for immunization of rabbits. The intravenous immunization schedule and conjugate preparation have been described previously. ${ }^{11}$ The fluoresceinto-protein ratios of all conjugates were in the range of 3.6 to $10.3 \mu \mathrm{g} / \mathrm{mg} .13$

Collection of SAMPLes.-Plaque samples.Samples of plaque were collected from multiple sites and pooled to give an approximal and an occlusal sample for each subject. ${ }^{14}$ 
The approximal sample was collected with the aid of dental floss from the surfaces between the premolars and from between the first and second molars of each quadrant. Occlusal fissure samples were obtained by means of a pointed wire. A separate wire $(1 \mathrm{~cm})$ held in the middle with a hemostat, was used to sample the fissures of a second premolar and second molar present in each quadrant. Each floss or wire was placed into $10 \mathrm{ml}$ of a reduced transport fluid (RTF). The samples were collected in the evening, and were refrigerated at $4 \mathrm{C}$ until they were cultured the next day. The samples then were sonically dispersed for 10 seconds, serially diluted, and $0.05 \mathrm{ml}$ aliquots from over a 1,000-fold dilution range plated in duplicate with an Eppendorf pipette onto MM10 sucrose agar. ${ }^{14}$ The plates were incubated at $37 \mathrm{C}$ in an anaerobic chamber for five to seven days and then used for the determination of total viable count and $S$ mutans count. In a separate procedure, a $0.05-\mathrm{ml}$ aliquot was taken from the collection tube and inoculated into a $0.1 \%$ Trypticase, $0.05 \%$ yeast extract, $0.1 \%$ mannitol broth (TYM broth). After overnight incubation the growth in the TYM broth was examined for $S$ mutans serotypes by the direct FAT. In addition, the plaque suspension in the collection tube was concentrated by centrifugation and smears were made of this sediment. Thus, each fissure and approximal sample was examined in three ways for $S$ mutans: serial dilution cultural method; smears of overnight growth of plaque examined by the direct FAT; and smears of the dispersed plaque examined by the direct FAT. The occlusal and approximal plaque samples were collected before and after topical treatment with acidulated-phosphate fluoride gel (APF). 14 Thus it was possible to monitor the efficacy of the APF in reducing the levels of $S$ mutans by the three methods described.

Saliva samples.-Unstimulated salivary samples were collected from children with ten or more carious surfaces immedately before and after one week of topical $5 \%$ kanamycin gel treatment. ${ }^{15}$ One-milliliter aliquots were added to $9 \mathrm{ml} \mathrm{RTF}$. The samples were cultured using the same dispersion, dilution, and inoculation methodology as described with the plaque, except that an additional medium, Mitis-Salivarius agar containing $20 \%$ sucrose and $0.2 \mu \mathrm{g}$ bacitracin/ ml16 but minus tellurite, also was inoculated. The samples were processed within two to four hours after collection. The salivary suspension in the collection tube was concentrated by centrifugation, and smears of the sediment were examined by the direct FAT.

Direct Fluorescent Antibody Test.Smears of dental plaque, overnight plaque growth in TYM broth, salivary sediment, or the $S$ mutans vaccines were incubated with the working titer of the conjugated antiserums in a moist chamber at room temperature for 30 minutes. The smears were washed and a $1: 20$ dilution of chelated eriochrome black was left on the smears for 10 to 15 seconds, after which the slides were rinsed with phosphate-buffered saline $(\mathrm{pH}$, 7.6) and mounted in buffered glycerine. The eriochrome black previously was shown to essentially eliminate nonspecific fluorescence and weak cross-reactions. ${ }^{11}$ The smears were examined with a Zeiss GFL microscope with an Osram $200 \mathrm{~W}$ mercury vapor arc lamp and a dark-field condenser. The optics consisted of a $\times 40$ oil immersion objective and $\times 12.5$ occulars. BG12 excitation and PG4/ GG4 barrier filters were used in combination. Only cells that exhibited a coccoid morphology and a $3^{+}$to $4^{+}$fluorescence were recorded as positive. In the smears made from the concentrated plaque and saliva all of the positive cells present in 20 to 30 high power fields were counted, so as to provide an estimate of the levels of $S$ mutans in the samples. The smears made from the overnicht plaoue cultures usually had too many cells to he enumerated, and the levels of $S$ mutans were simply scored as absent, few, or too numerous to count.

\section{Results}

One or more serotypes of $S$ mutans were found by direct FAT in the overnight broth cultures of approximal and occlusal plaque (Table 1). Examination of the plaque smears showed $S$ mutans to be present in $100 \%$ of the approximal samples and in $61 \%$ of the occlusal samples. This latter percentage reflected an insufficient number of cells available for microscopic examination in some occlusal samples. The culture studies showed $S$ mutans to be present in $72 \%$ of the approximal samples and $88 \%$ of the occlusal samples. $S$ mutans averaged about $1 \pm 0.4 \%$ of the total viable count in the approximal 
TABLE 1

Frequency of Detection of $S$ mutans by Direct FAT OR By Cultural METhods in Either approximal or Occlusal plaque

\begin{tabular}{|c|c|c|c|}
\hline \multirow[b]{3}{*}{ Plaque } & \multicolumn{3}{|c|}{ Percent Positive Detection } \\
\hline & \multicolumn{2}{|c|}{ FAT } & \multirow[b]{2}{*}{$\begin{array}{c}\text { Cultura } \\
\text { Serial } \\
\text { Dilution } \\
\text { Method }\end{array}$} \\
\hline & $\begin{array}{l}\text { Plaque } \\
\text { Smears }\end{array}$ & $\begin{array}{c}\text { Overnight } \\
\text { Broth } \\
\text { Growth }\end{array}$ & \\
\hline Approximal & 100 & 100 & 72 \\
\hline Occlusal & 61 & 100 & 88 \\
\hline
\end{tabular}

samples, and about $9 \pm 3.0 \%$ in the occlusal samples. ${ }^{14}$

The smears made of the dispersed plaque samples showed serotype $d$ to be the most common serotype present. Serotype $d$ was found in $96 \%$ of the approximal plaques and in $51 \%$ of the occlusal plaques (Table 2). Serotype $b$ was found in $57 \%$ of the approximal plaques, but only rarely detected in the occlusal plaques. Serotype $c$ and $e$ cells were not evident in any of the plaque smears. A different profile appeared when the overnight plaque growth in TYM broth was examined. Serotype $c$ was found in all approximal plaques and in $88 \%$ of the occlusal plaques (Table 2). Serotype $e$ was also a predominant type; it was present in about $75 \%$ of both plaques. Serotype $d$ was still commonly found, but serotype $b$ was greatly reduced in occurrence. Clearly there was a discrepancy with regard to the pres- ence of serotype $c$ in the smears made directly from the plaque and the overnight broth growth of the same plaque. This can be explained in at least two ways. The first explanation is that serotype $c$ was present in such low numbers in the plaque that it could not be detected by the direct FAT. However, when this plaque was placed in broth, serotype $c$ was able to grow out rapidly so that now it was readily detected by the serotype $c$ conjugate. The alternate possibility is that serotype $c$ was present in the plaque in detectable numbers, but was coated in such a manner by plaque constituents that it could not be stained by the FAT reagents. After growth in broth, the various masking agents (that is, immune globulins and salivary glycoproteins) were diluted out, and the serotype $c$ cells were then stainable. These possibilities were tested in a separate experiment by randomly choosing $S$ mutans colonies from high dilution primary isolation plates and subculturing them directly into Trypticase soy broth. Over 100 such isolates were obtained from either MMl0 sucrose $^{7}$ or MSB16 plates and were shown to be mannitol-positive strains. They then were characterized using the biochemical scheme for $S$ mutans serotypes proposed by Shklair and Keene.12 Because these isolates would reflect the predominant cultivable serotypes of $S$ mutans, they would not contain serotype $c$ cells if the first explanation were true, but would if serotype $c$ cells were masked in the plaque, as proposed in the second ex-

TABLE 2

Relative Frequency of $S$ mutans Serotypes in Plaque Smears or in Broth Inoculated with Plaque as Determined by Direct Fluorescent ANTIbody Test

\begin{tabular}{|c|c|c|c|c|}
\hline \multirow[b]{2}{*}{ Samples } & \multicolumn{4}{|c|}{$\begin{array}{l}\text { Serotype Strains and } \\
\text { Working Titers } \\
\text { (no. of positive samples) }\end{array}$} \\
\hline & $\begin{array}{c}b \\
\text { (FA-1 } \\
\text { undi- } \\
\text { luted) }\end{array}$ & $\begin{array}{c}c \\
(\text { GS-5 } \\
1: 4)\end{array}$ & $\underset{(\mathrm{OIHI}}{d}$ & $\begin{array}{c}e \\
\text { (LM7 } \\
\text { undi- } \\
\text { luted) }\end{array}$ \\
\hline \multicolumn{5}{|l|}{ Pooled approximal plaque } \\
\hline Smear & 59 & $\mathbf{0}$ & 100 & 24 \\
\hline Broth & 11 & 48 & 86 & 35 \\
\hline \multicolumn{5}{|l|}{ Pooled occlusal plaque } \\
\hline Smear & 1 & $\mathbf{0}$ & 53 & 0 \\
\hline Broth & 8 & 42 & 60 & 36 \\
\hline Total samples (\% positive) & 104 & 48 & 104 & 48 \\
\hline Smears & 57 & 0 & 96 & 50 \\
\hline Broth & 11 & 100 & 83 & 75 \\
\hline
\end{tabular}

Note: -20 to 30 high-power fields counted. 
planation. Seventy-nine percent of the isolates were serotype $c$ strains, $5 \%$ were serotype $d$ strains, and $16 \%$ were serotype $e$ strains (Table 3). Thirty-five serotype $c$ strains were separately tested with the serotype $c$ conjugate and 32 of those gave a $3^{+}$ or $4+$ FAT reaction.

The FAT on the overnight broth cultures provided no information on the levels of $S$ mutans in the plaque. For this reason, this procedure was abandoned as a means of monitoring whether the APF or placebo gels had an effect on the levels of $S$ mutans. Concurrent cultural studies showed that APF treatment caused a persistent 45 to $75 \%$ reduction in the percentages of $S$ mutans in the occlusal samples. ${ }^{14}$ The number of occlusal plaques that exhibited change or no change in $S$ mutans levels as a result of treatment is presented in Table 4 . Both the FAT of plaque smears and the cultural data showed the APF to decrease the levels of $S$ mutans in 11 to 14 plaque samples. In the placebo group, the culture data demonstrated that $S$ mutans increased in 12 of 21 samples, whereas the FAT data showed a decrease in levels of $S$ mutans. Some of the FAT on the plaques were complicated by the low number of bacterial cells present in the samples.

The recent description of a selective medium for $S$ mutans ${ }^{16}$ suggested that this organism could be quantitated reliably when present in low numbers in a sample such as saliva. Accordingly, the levels of $S$ mutans in saliva were determined by both fluorescent microscopy and culture means. These studies were done in conjunction with a clinical trial evaluating the ability of a $5 \%$

\section{TABLE 3}

Distribution of $S$ Mutans Serotypes in Randomly Chosen Golonies Obtained From HIGH DILUTION Primary IsOlation Plates

\begin{tabular}{lcccc}
\hline & \multicolumn{3}{c}{ Serotypes* } & \\
\cline { 2 - 4 } \multicolumn{1}{c}{ Source } & $c$ & $d$ & $e$ & Totals \\
\hline $\begin{array}{l}\text { Schoolchildren } \\
\begin{array}{l}\text { Pedodontic } \\
\quad \text { patients }\end{array}\end{array}$ & 22 & 4 & 12 & 38 \\
$\begin{array}{l}\text { Children with } \\
\quad \text { rampant-caries }\end{array}$ & 42 & 0 & 2 & 23 \\
\begin{tabular}{l} 
Totals \\
\hline
\end{tabular} & 85 & 5 & 18 & 108 \\
\hline
\end{tabular}

- Determined using biochemical criteria of Shklair and Keene. ${ }^{13}$
TABLE 4

Number of Fissure plaque Samples Exhibiting Change or No Change in Levels of $S$ mutans Following Fluoride or Placebo Treatment

\begin{tabular}{lcrr}
\hline & \multicolumn{2}{c}{ FAT of } & \\
\cline { 2 - 3 } Preatment & $b$ & $d$ & Culture \\
\hline Fluoride & & & \\
Increased & 3 & 3 & 5 \\
Decreased & 14 & 11 & 11 \\
No change & 0 & 0 & 0 \\
Placebo & & & \\
Increased & $\ldots$ & 1 & 12 \\
Decreased & 1 & 14 & 8 \\
No change & $\ldots$ & 3 & 1 \\
$\quad$ Total & & & 21 \\
Difference & & & \\
$\quad$ (increase minus decrease) & & -6 \\
Fluoride & -11 & -8 & +4 \\
Placebo & -1 & -13 & \\
\hline
\end{tabular}

Note: Serotypes $c$ and $e$ were not clear on smears.

kanamycin or placebo gel to lower or eliminate $S$ mutans from the tooth surfaces of children diagnosed as having rampant caries (that is, $>10$ carious surfaces). The $S \mathrm{mu}$ tans levels immediately before and immediately after one week of gel treatment were determined. The data were expressed as the number of saliva samples in which $S$ mutans increased, decreased, or did not appear to change as a result of treatment (Table 5). Kanamycin decreased the levels of $S$ mutans in most of the children, whereas these levels tended to increase in the placebo children. This pattern was seen both by the FAT and culture procedures. Serotypes $c$ and $e$ were discernible in the salivary smears, whereas previously they were rarely encountered in the plaque smears.

\section{Discussion}

Serotype $c$ is reported to be the most prevalent serotype in plaque based on FATs performed on plaque cultures after growth in various broths $s^{5,8}$ or on FAT and biochemical reactions of plaque isolates.9.10.12 When our plaques were inoculated into broth, serotype $c$ was found in all the samples, despite the fact that in the direct examination of the plaque, this serotype could rarely be identified. Subsequent testing of recent isolates of $S$ mutans obtained from high dilution plates showed that the overwhelming majority of them were serotype $c$ strains. Serotype $d$ strains, which were so common in the direct 
TABLE 5

EFfect of Kanamycin or Placebo Treatment on levels of S Mutans SERotypes in Saliva

\begin{tabular}{|c|c|c|c|c|c|}
\hline & \multicolumn{4}{|c|}{ Serotypes } & \multirow[b]{2}{*}{ Culture } \\
\hline & $b$ & $c$ & $d$ & $e$ & \\
\hline \multicolumn{6}{|l|}{ Kanamycin } \\
\hline Increased & 2 & 2 & 2 & 1 & 1 \\
\hline Decreased & 4 & 4 & 5 & 5 & 8 \\
\hline No change & 3 & 4 & 2 & 1 & 3 \\
\hline \multicolumn{6}{|l|}{ Placebo } \\
\hline Increased & 5 & 6 & 7 & 6 & 5 \\
\hline Decreased & 3 & 4 & 6 & 4 & 2 \\
\hline No change & 5 & 3 & 2 & 2 & 1 \\
\hline \multicolumn{6}{|c|}{$\begin{array}{l}\text { Difference } \\
\quad \text { (increase minus decrease) }\end{array}$} \\
\hline Kanamycin & -2 & -2 & -3 & -4 & -7 \\
\hline Placebo & +2 & +2 & +1 & +2 & +3 \\
\hline
\end{tabular}

Note: Direct FAT on salivary sediment.

smears, accounted for $5 \%$ of the isolates. This would indicate that serotype $c$ is in plaque in high numbers, but was not stained by the conjugates used, even though these conjugates were adequate to give specific reactions with in vitro grown serotype $c$ strains. This result can be interpreted as indicating that the serotype $c$ FAT-reactive sites are masked in the direct plaque samples by salivary glycoproteins, glucans, or immunoglobulins. The fact that only serotype $c$ cells seem to be affected would suggest some type of specific masking agent such as immunoglobulins.

The possibility that plaque contains cocci in high numbers which cross react with the anti- $d$ serum would have to be eliminated as a cause for the high levels of serotype $d$ detected. The presence of serotype $b$ cells in plaque smears probably can be accounted for by cross-reactions of this antiserum with teichoic acid containing streptococci. ${ }^{17}$

This investigation raises questions about the future value of the FAT in showing a relationship between $S$ mutans and human decay. FATs performed on broth cultures of plaque may be quick and adequate for the detection of $S$ mutans in the sample.4 However, these tests show serotype $c$ and may not demonstrate the other serotypes present in the plaque. And more importantly, they provide no quantitative information concerning the proportions or levels of the various serotypes in the plaque samples. Quantitative information is necessary, because culture studies indicate that many cariesfree individuals have detectable $S m u$ tans in their saliva and in pooled plaque samples obtained from retentive surfaces (Table 6) . ${ }^{7}$ If plaque removed from a single occlusal fissure is cultured, one of three $S$ mutans positive samples comes from a clinically cariesfree surface (Table 6). Thus, if the FAT for $S$ mutans is designed to only detect $S$ mutans, the information obtained may not have much diagnostic value. The culture studies demonstrate a highly significant relationship between the levels or proportions of $S$ mutans and caries activity (that is, Kruskall Wallis rank test $P<0.01$ ) . ${ }^{7}$ For this reason we chose to make the FAT as quantitative as possible by enumerating the levels of the various serotypes in plaque smears. This required the selection of samples that contained enough cells to be observed under the microscope and thereby eliminated the examination of plaques removed from single fissure sites because they have only from 0.01 to $18 \times 10^{6}$ cells. Thus, the plaque most associated with the disease process could not be studied. Pooled plaque samples obtained from retentive surfaces contain from 2 to $111 \times 10^{6}$ cells per sample and can usually be counted by microscopy, as was done to obtain the data shown in Tables 1, 2, and 4. However, when small numbers are encountered, they cannot be reliably examined by FAT. Hence, samples containing more cells (such as pooled plaque obtained from smooth and retentive surfaces and saliva) should be used in a quantitative FAT. Saliva contains about 200 to $300 \times$ 
TABLE 6

Relationship Between Proportional levels of S mutans in Saliva, Pooled Plaque, and Single-Site Plaque and Clinic diagnosis of Carifs

\begin{tabular}{|c|c|c|c|c|}
\hline \multirow[b]{2}{*}{ Sample } & \multicolumn{2}{|c|}{$S$ mutans } & \multicolumn{2}{|c|}{$\begin{array}{l}S \text { mutans } \\
\text { account for }\end{array}$} \\
\hline & $\begin{array}{l}\text { Not De- } \\
\text { tected }\end{array}$ & Detected & $\begin{array}{l}\text { I- } 10 \% \\
\text { of CFU }\end{array}$ & $\begin{array}{l}>10 \% \\
\text { of CFU }\end{array}$ \\
\hline \multicolumn{5}{|l|}{ Salivat } \\
\hline Cariesfree & 9 & 42 & 5 & 0 \\
\hline$>5$ decayed teeth & 0 & 53 & 23 & 0 \\
\hline \multicolumn{5}{|l|}{ Pooled Plaquet } \\
\hline Cariesfree & 11 & 16 & 2 & 8 \\
\hline Rampant caries & 6 & 37 & 8 & 28 \\
\hline \multicolumn{5}{|l|}{ Single Fissure } \\
\hline Cariesfree & 70 & 53 & 17 & 26 \\
\hline Carious & 30 & 100 & 32 & 65 \\
\hline
\end{tabular}

\footnotetext{
Note: Adapted from data presented by Loesche et al.?

* CFU is colony forming units on either MM10 sucrose or Mitis-Salivarius agar with $20 \%$ sucrose and bacitracin.

t Relationship between increasing levels of $s$ mutans and caries activity is significant $P<0.001$, Kruskall Wallis rank.
}

$10^{6}$ cells per $\mathrm{ml}$, an amount that can easily be seen under the microscope. The cultural and FAT examination of saliva before and after kanamycin or placebo treatment showed similar directional changes in the levels of $S$ mutans, indicating that both procedures were demonstrating a reduction in $S$ mutans level by the kanamycin treatment (Table 5). These quantitative FATs required the counting of 20 to 30 microscopic fields of each sample for each of the five serotypes. This required a considerable amount of time and antiserums. If certain serotypes, such as serotype $c$, cannot be visualized in these smears, and this serotype is the dominant one in plaque, then the FAT on plaque samples provides inadequate qualitative information. Because the FATs performed on broth do not provide quantitative information, it appears at this time that the exclusive use of FATs for diagnosis of $S m u$ tans in oral samples is premature.

\section{References}

I. LoEsche, W.J.: Dental Infections, in Balows, A.; DehaAn, R.M.; Guze, L.B.; and Dowell, V.R. (eds): Anaerobic Bacteria, Springfield: Charles C Thomas, 1974, pp. 409-434.

2. Zinner, D., and JABLoN, J.: Human Streptococcal Strains in Experimental Caries, in HARRIS, R.S. (ed) : The Art and Science of Dental Caries Research, New York: Academic Press, 1968, pp 87-109.

3. BRatThall, D.: Immunofluorescent Identification of Streptococcus mutans, Odontol Revy 23: 1-20, 1972.
4. DUANY, L.F.; JAbLon, J.M.; and ZiNnER, D.D.: Epidemiologic Studies of Caries-Free and Caries-Active Students. I. Prevalence of Potentially Cariogenic Streptococci, J Dent Res 51: 723-726, 1972.

5. BRatthall, D.: Demonstration of Strepto. coccus mutans Strains in Some Selected Areas of the World, Odontol Revy 23: 40I-410, 1972.

6. De Stoppelaar, J.D.; Van Houte, J.; and Backer Dirks, O.: The Relationship Between Extracellular Polysaccharide-Producing Streptococci and Smooth Surface Caries in 13-year-old children, Caries Res 3: 190 . $199,1969$.

7. LOEsche, W.J.; Rowan, J.; Straffon, L.H.; and Loos, P.J.: Streptococcus mutans and Human Dental Decay, submitted for publication.

8. Thomson, L.A.; Little, W.; and Hageage, G.J.: Application of Fluorescent Antibody Methods in the Analysis of Plaque Samples, $J$ Dent Res 55 (Special Issue A) : A80-A86, 1976.

9. Perch, B.; KJems, E.; and Ravi, T.: Biochemical and Serological Properties of Streptococcus mutans from Various Human and Animal Sources, Acta Pathol Microbiol Scand (Section 3) 82: 357-370, 1974.

10. Hamada, S., and Ooshima, T.: Production and Properties of Bacteriocins (Mutacins) from Streptococcus mutans, Arch Oral Biol, in press.

11. Grenier, E.M.; Eveland, W.C.; and Loesche, W.J.: Identification of Streptococcus mutans Serotypes in Dental Plaque by Fluorescent Antibody Techniques, Arch Oral Biol 18: 707-715, 1973. 
12. ShKLAIR, I.L., and KEENE, H.J.: A Biochemical Scheme for the Separation of the Five Varieties of Streptococcus mutans, Arch Oral Biol 19: 1079-1082, 1974.

13. Wells, P.F.; Miller, C.E.; and NAdEL, M.K.: Rapid Fluorescein and Protein Assay Method for Fluorescent Antibody Conjugates, $A p p l$ Microbiol 14: 271-275, 1966.

14. Loesche, W.J.; SYed, S.A.; Murray, R.J.; and MellberG, J.R.: Effect of Topical Acidulated Phosphate Fluoride on Percentage of Streptococcus mutans and Streptococcus sanguis in Plaque. II. Pooled Occlusal and
Pooled Approximal Samples, Caries Res 9: 139-155, 1975.

15. Loesche, W.J.; Bradbury, D.R.; and WoolFOlk, M.P.: Effect of Topical Kanamycin Gel on the Levels of Streptococcus mutans in Rampant Caries Subject, $J$ Dent Res 54 (Special Issue A) : Abstract No. 335, 1975.

16. Gold, O.C.; Jordan, H.V.; and VAN HOUTE, J.: A Selective Medium for Streptococcus mutans, Arch Oral Biol 18: 1357-1364, 1973.

17. KNOX, K.W; and WICkEN, A.J.: Grouping and Cross-Reacting Antigens of Oral Lactic Acid Bacteria, J Dent Res 55 (Special Issue A) : A116-A122, 1976. 